WINPEC Working Paper Series No.E1805

July 2018

\title{
The Unbinding Core for Coalitional Form Games
}

Takaaki Abe, Yukihiko Funaki

Waseda INstitute of Political EConomy

Waseda University

Tokyo,Japan 


\title{
The Unbinding Core for Coalitional Form Games*
}

\author{
Takaaki Abe ${ }^{\dagger} \quad$ Yukihiko Funaki ${ }^{\ddagger}$
}

May, 2018

\begin{abstract}
In this paper, we introduce a new concept of core by extending the definition of deviation. The traditional definition of deviation allows for players to deviate if some profitable allocation exists after their deviation, while our new definition requires that all possible allocations are profitable. Hence, our core becomes a superset of the traditional core. We examine some properties that our new core satisfies and provide a sufficient condition for being nonempty. Moreover, we apply Ray's (1989) credibility to our core.
\end{abstract}

Keywords: cooperative game, core, credibility, deviation

JEL Classification: C71

\section{Introduction}

The traditional theory of core is based on the assumption that if members of a deviating coalition choose a particular allocation, then they can implement the allocation with certainty. The mathematical definition of deviation exhibits this spirit. Let $S$ be a coalition of players, $\mathbb{R}^{S}$ be the set of $|S|$-dimensional real vectors, where $|S|$ is the number of members of $S$, and $V(S) \subseteq \mathbb{R}^{S}$ be the set of feasible allocations for $S$. A coalition $S$ deviates from an allocation $x$ if there exists $y \in V(S)$ such that $y_{j}>x_{j}$ for any $j \in S$, namely, if there exists at least one profitable outcome $y$, they can deviate and obtain $y$ with certainty. The traditional core is defined by the set of allocations from which no coalition deviates. However, there are many actual economic scenes in which the members of a coalition cannot determine or unanimously choose an allocation to deviate. To see this, we consider the following two cases: external uncertainty and internal uncertainty.

We begin with external uncertainty. We consider $n$ players who work for a firm. Let $N$ be the set of the players. Each player receives a payoff $\frac{1}{n}$ by working for the firm. Now, some players try to become independent from the firm $N$ and start their own firm $S \subsetneq N,|S|>\frac{n}{2}$, where $|S|$ is number of members of $S$. If the economy is good, each member of $S$ obtains $\frac{1}{|S|}$. If the economy is bad, they obtain zero.

\footnotetext{
* The authors thank Youngsub Chun, Stéphane Gonzalez, Toru Hokari, Fuhito Kojima, Peter Sudöhlter, and the seminar participants of SING 13 and EAGT 2017 for constructive comments. Takaaki Abe acknowledges financial support from the Japan Society for the Promotion of Science (JSPS).

$\dagger$ JSPS Research Fellow. Graduate School of Economics, Waseda University. 1-6-1, Nishi-waseda, Shinjuku-ku, Tokyo 169-8050, Japan. E-mail: takatomo3639@asagi.waseda.jp

¥ School of Political Science and Economics, Waseda University. 1-6-1, Nishi-Waseda, Shinjuku-ku, Tokyo 169-8050, Japan. E-mail: funaki@waseda.jp
} 
We formulate the set of possible allocations for $S$ as $V(S)=\left\{\left(\frac{1}{|S|}\right)_{j \in S},(0)_{j \in S}\right\} \subseteq \mathbb{R}^{S}$ if $|S|>\frac{n}{2}$, and $V(S)=\left\{(0)_{j \in S}\right\}$ otherwise. As mentioned above, the traditional definition may allow for coalition $S$ to deviate from $N$ because the good outcome $\left(\frac{1}{|S|}\right)_{j \in S}$ exists, such that $\frac{1}{|S|}>\frac{1}{n}$ for every member of $S$ with $|S|>\frac{n}{2}$. Certainly, if the outcomes in $V(S)$ are derived from controllable factors, e.g., quantity of input or the technology they employ, then this definition might be plausible. However, as described in the example, the condition of the economy is not controllable for the members of $S$, and hence, $S$ cannot "choose" the good outcome. Therefore, $S$ might not deviate from $\left(\frac{1}{n}\right)_{j \in N}$, as its members are afraid of $(0)_{j \in S}$.

Coalition $S$ 's external uncertainty may be caused by the other players $N \backslash S$. The class of partition function form games or games with externalities (Thrall 1961; Thrall and Lucas 1963) is a seminal attempt that tries to model this kind of external uncertainty. For a partition function form game, the set of allocations of a coalition $S$ depends on $S$ 's members and the structure of $N \backslash S$, which is mathematically defined as a partition of $N \backslash S$. Of course, the members of $S$ cannot choose the partition of $N \backslash S$. Therefore, each coalition $S$ has uncertainty about their allocations. A coalition function form game can be thought of as a model to avoid this uncertainty. Aumann (1960) assumes that $V(S)$ is the set of feasible allocations that are independent of conditions outside $S$. This is justified as follows: $V(S)$ is the set of "maximin" allocations that $S$ can guarantee given that $N \backslash S$ minimizes it. In other words, the problem of external uncertainty has been avoided by "fixing" uncertainty to the worst outcome. Hence, if we do not fix an external partition, the definition of core becomes plural. ${ }^{* 1}$

Uncertainty is also found inside a coalition $S$ that is deviating from $N$. Now, let $S=\{i, j\}$ and suppose that if the members of $S$ become independent from $N$ and start their own business, then they obtain total surplus 1 with certainty. Moreover, suppose that player $i$ is an egalitarian and claims 0.5 as his share (therefore, 0.5 for $j$ ), while player $j$ is a performance-based player and actually contributes more than $i$; she claims 0.6 (thus 0.4 for $i$ ). Their preferences then conflict within $S$, as $i$ prefers $(0.5,0.5)$, but $j$ prefers $(0.4,0.6)$. As this example illustrates, conflict between deviating players may prevent them from reaching a binding agreement, which may not resolve the traditional definition of deviation and may admit of an alternative formulation of core.

Internal uncertainty also appears in internal conflict for coordination games and equilibrium selection. To see this, we consider the following simple stag hunt game.*2 Each of three hunters $A, B, C$ chooses his (pure) strategy stag or hare. If three hunters choose stag, then they get a large stag and obtain a total payoff of 21 . If two hunters choose stag, they obtain a middle stag and a total payoff of 16 . If one hunter chooses stag, he cannot hunt any stag and obtains zero. If he chooses hare alone, he gets one hare and a payoff of 2, independent of the others' choices. These three hunters first agree to form a team, or a coalition, and then go hunting, in which each of them individually chooses stag or hare. If a three-hunter team is formed, then the corresponding payoff matrix of the three-hunter game

\footnotetext{
${ }^{* 1}$ Funaki and Yamato (1999) consider the pessimistic core and the optimistic core for partition function form games. Kóczy (2007) defines a recursive core for partition function form games. Abe and Funaki (2017) propose conditions for the cores to be nonempty. Abe (2018) characterizes the cores by consistency properties in the presence of externalities.

*2 Stag hunt games are not the only examples illustrating internal conflict. Almost all coordination games contain a similar conflict; another example is battle of the sexes.
} 
is as summarized in Table 1 . Table 2 is that of a two-hunter team. If one hunter goes alone, then he gets one hare and obtains a payoff of 2. Clearly, for each of three-hunter and two-hunter teams, there are two Nash equilibria: (stag,stag,stag), (hare,hare,hare); and (stag,stag), (hare,hare). As Harsanyi and Selten (1988) discuss, the stag-equilibrium payoff dominates the hare-equilibrium, and the latter risk dominates the former. Now, which team of hunters is formed and goes hunting? Moreover, which outcome (namely, payoffs and strategy profile) is chosen by the hunters? If members of a two-hunter team agree on the stag-equilibrium and can bind each other to the equilibrium, then allocation $(7,7,7)$ of the stag-equilibrium should be blocked by the coalition of the two hunters through allocation $(8,8)$. However, as the theory of equilibrium selection questions, it is not necessarily obvious that each of the two hunters commits to accomplish the stag-equilibrium. This uncertainty is also a background that we attempt to define for a new core concept.

Table 1 Stag hunt of a three-hunter team

\begin{tabular}{|c|c|c|c|c|c|c|c|}
\hline & \multirow[b]{2}{*}{ C: stag } & \multicolumn{2}{|c|}{ B } & \multirow{2}{*}{\multicolumn{2}{|c|}{ C: hare }} & \multicolumn{2}{|c|}{ B } \\
\hline & & stag & hare & & & stag & hare \\
\hline \multirow{2}{*}{ A } & stag & $7,7,7$ & $8,6,8$ & \multirow{2}{*}{ A } & stag & $8,8,6$ & $0,2,2$ \\
\hline & hare & $6,8,8$ & $2,2,0$ & & hare & $2,0,2$ & $2,2,2$ \\
\hline
\end{tabular}

Table 2 Stag hunt of a two-hunter team

\begin{tabular}{c|cc} 
& stag & hare \\
\hline stag & 8,8 & 0,2 \\
hare & 2,0 & 2,2
\end{tabular}

Given these arguments, how should we deal with uncertainty and modify the concept of core? This question motivates us to introduce a new notion of core, the unbinding core. This is our main purpose of the paper. We also investigate important properties of the unbinding core that the original core obeys. We introduce this new concept of core and analyze some numerical examples, including the stag hunt game, in Section 2. In Section 3, we present general basic differences and similarities between the core and the unbinding core. In Section 4, we propose a sufficient condition for the unbinding core to be nonempty. In Section 5, we consider credibility of the unbinding core. The notion of credible core was introduced by Ray (1989) to formulate a deviation by credibility. The credible (traditional) core coincides with the traditional core, while this coincidence does not hold for the unbinding core. Section 6 presents concluding remarks.

\section{Preliminaries}

Let $N=\{1, \ldots, n\}$ be the set of players. A coalition $S$ is a subset of $N: S \subseteq N$. We denote by $|S|$ the number of players in $S$ and use $n$ to denote $|N|$. For any nonempty coalition $S \subseteq N$, let $V(S)$ 
denote the set of all possible allocations for $S$, namely, $V(S) \subseteq \mathbb{R}^{S}$. We assume that $V(S) \neq \emptyset$ for any $S \subseteq N$. If allocations in $V(S)$ are countable, we denote by $|V(S)|$ the number of allocations in $V(S)$. For example, if $V(\{1,2\})=\{(6,4),(5,5),(4,6)\}$, then $|V(\{1,2\})|=3$. An outcome is a pair of an allocation and a coalition structure. A coalition structure of $N$ is a partition of $N$. We denote it by $\mathcal{P}$ and the set of all partitions of $N$ by $\Pi(N)$. Let $F \subseteq \mathbb{R}^{N}$ be the set of outcomes, formally, $F=\left\{(x, \mathcal{P}) \mid \mathcal{P} \in \Pi(N), x \in \mathbb{R}^{N}, x^{S} \in V(S)\right.$ for any $\left.S \in \mathcal{P}\right\}$, where $x^{S}$ is a restriction of $x$ to $S$, namely $x^{S} \in \mathbb{R}^{S}$ and $x_{j}^{S}=x_{j}$ for any $j \in S$. A game is $(N, F, V)$. In this paper, we assume the formation of the grand coalition and restrict our attention to the stability of allocations for it. Hence, we assume that $F=\{(x,\{N\}) \mid x \in V(N)\}$. We use $(N, V)$, or simply $V$, to denote a game.

A coalition $S$ deviates from an allocation $x \in \mathbb{R}^{N}$ if there exists $y \in V(S)$ such that $y_{j}>x_{j}$ for any $j \in S .^{* 3}$ A coalition $S$ unbindingly deviates from an allocation $x \in \mathbb{R}^{N}$ if for all $y \in V(S), y_{j}>x_{j}$ for any $j \in S$. By $S \operatorname{dev} x\left(S \operatorname{dev}^{\mathrm{UB}} x\right)$, we denote a coalition $S$ 's deviation (unbinding deviation) from an allocation $x$. The unbinding deviation illustrates deviating players' cautious attitude: players do not deviate from $N$ unless all possible outcomes are profitable.

We define the core $C$ as the set of allocations in $V(N)$ from which no coalition deviates, formally,

$$
C(V)=\{x \in V(N) \mid \nexists S \subseteq N \text { such that } S \operatorname{dev} x\} .
$$

Similarly, the unbinding core $C^{\mathrm{UB}}$ is defined by

$$
C^{\mathrm{UB}}(V)=\left\{x \in V(N) \mid \nexists S \subseteq N \text { such that } S \mathrm{dev}^{\mathrm{UB}} x\right\}{ }^{* 4}
$$

Clearly, $S \operatorname{dev}^{\mathrm{UB}} x \Rightarrow S \operatorname{dev} x$. Hence, for every $V$, we have $C(V) \subseteq C^{\mathrm{UB}}(V)$, which illustrates that conflicts and uncertainty make players less likely to deviate. Therefore, if $|V(S)|=1$ for every $S \subsetneq N$, then we have $C^{U B}(V)=C(V)$.

Example 2.1. We now analyze the stag hunt game introduced in Section 1 . We adopt Nash equilibria as outcomes and set $V$ as follows. ${ }^{* 5}$

$$
V(S)= \begin{cases}\{(7,7,7),(2,2,2)\} & \text { if }|S|=3, \\ \{(8,8),(2,2)\} & \text { if }|S|=2, \\ \{(2)\} & \text { if }|S|=1 .\end{cases}
$$

We first focus on the traditional core. The core is empty. This is interpreted as follows: both allocations for the three-hunter team, $(7,7,7)$ and $(2,2,2)$, are deviated by each two-hunter team through $(8,8)$, which means that each team consisting of two hunters is binding and the two hunters can achieve the stag-equilibrium with certainty. This brings about the empty core. Now, we consider the unbinding core. In the view of unbinding deviation, the members of a team cannot bind each other to a particular

*3 There are various definitions for deviation. This definition is the one employed by, for example, Ray (1989) and Peleg and Sudhölter (2007) (for the latter, see Subsection 12.1: "The Core of NTU Games"). Another definition is discussed in Section 6.

${ }^{* 4}$ If we do not assume the formation of the grand coalition, the definitions are as follows: $S \operatorname{dev}(x, \mathcal{P})$ if $\exists y \in V(S)$ s.t. $y_{j}>x_{j} \forall j \in S$, and $C(V)=\{(x, \mathcal{P}) \in F \mid \nexists S \subseteq N$ s.t. $S \operatorname{dev}(x, \mathcal{P})\}$. The unbinding core is also defined in the same manner. These general settings are desirable in the context of coalition formation and games with externalities. See, for example, Greenberg (1994) and Kóczy and Lauwers (2004).

*5 It is rational for the member of a one-hunter team, namely, a hunter, to choose hare and obtain payoff 2 because if he chooses stag alone, he obtains zero. 
outcome: once they form a team and go hunting, each hunter makes a decision individually and plays a stag hunt game. Neither $(7,7,7)$ nor $(2,2,2)$ are eliminated by any two-hunter deviation (or any onehunter deviation) because hunters are cautious of the possibility that their deviation may lead to the hare-equilibrium $(2,2)$, which is not profitable for them. Moreover, even the grand coalition does not deviate from $(2,2,2)$ to $(7,7,7)$ in the view of unbinding deviation because the three hunters cannot bind each other to the good equilibrium. ${ }^{* 6}$ As a consequence, $C^{\mathrm{UB}}(V)=\{(7,7,7),(2,2,2)\} .{ }^{* 7}$ It may be worth mentioning that their cautious attitude maintains the formation of the grand coalition as a result.

Example 2.2. We consider a variant of the three-person majority game: $N=\{1,2,3\}$,

$$
\begin{aligned}
V(N) & =\left\{x \in \mathbb{R}^{3} \mid \sum_{j \in N} x_{j}=1\right\}, \\
V(\{1,2\}) & =\{(0.5,0.5)\} \\
V(\{1,3\}) & =\{(0.5,0.5)\} \\
V(\{2,3\}) & =\{(0.5,0.5),(0.6,0.4),(0.4,0.6)\}, \\
V(\{i\}) & =\{(0)\} \text { for all } i \in N .
\end{aligned}
$$

In this example, for any $x \in V(N) \backslash\{(0,0.5,0.5)\}$, there exists a coalition $S$ such that $S \operatorname{dev} x$. Hence,

$$
C(V)=\{(0,0.5,0.5)\} \text {. }
$$

In contrast, the unbinding core becomes a proper superset of the core. For any set $X$, we denote the convex hull of $X$ by $c o(X)$ (including extreme points) and the interior of $X$ by $\operatorname{int} X$. In addition to the allocation $(0,0.5,0.5)$, allocations $(0.5,0,0.5)$ and $(0.5,0.5,0)$ are also the members of the unbinding core. Moreover, define $L_{a}:=c o(\{(0.5,0,0.5),(0.6,0,0.4)\}), L_{b}:=\operatorname{co}(\{(0.5,0.5,0),(0.6,0.4,0)\}), A_{a}:=$ $\operatorname{intco}(\{(0.5,0,0.5),(0.6,0,0.4),(0.5,0.1,0.4)\})$ and $A_{b}:=\operatorname{intco}(\{(0.5,0.5,0),(0.6,0.4,0),(0.5,0.4,0.1)\})$. We have

$$
C^{\mathrm{UB}}(V)=\{(0,0.5,0.5)\} \cup L_{a} \cup L_{b} \cup A_{a} \cup A_{b} .
$$

The expanded allocations in $L_{a} \cup A_{a}$ are those that players 1 and 2 derive from player 3 through the presence of $(0,0.6,0.4)$. Once an allocation in $L_{a} \cup A_{a}$ results, allocation $(0,0.6,0.4)$ is no longer profitable for player 3 . Therefore, player 3 does not agree with player 2 to jointly deviate to form $\{2,3\}$, and it belongs to the unbinding core. The reason behind this disagreement lies in the multiplicity of allocations that the coalition $\{2,3\}$ has. This example tells us that uncertainty due to the small change in the possible allocations of coalition $\{2,3\}$ implies that it is possible for both players 2 and 3 to obtain benefit 0.1 because $(0.5,0.1,0.4)$ and $(0.5,0.4,0.1)$ are in the unbinding core.

If the set of allocations for $\{2,3\}$ is restricted to $V(\{2,3\})=\{(0.5,0.5)\}$, then every allocation in $L_{a} \cup A_{a}$, except for $(0.5,0,0.5)$, is bindingly and unbindingly deviated, and $C^{\mathrm{UB}}(V)=C(V)$. This can be seen as an example illustrating the relationship between the expansion of the unbinding core and that of $V(S)$. We elaborate upon this in the following section.

\footnotetext{
${ }^{* 6}$ Note that we do not force the deviating hunters to choose an equilibrium but simply let them take into account a wider range of possible outcomes that may happen after their deviation.

${ }^{* 7}$ It is not necessarily essential that allocation $(2,2,2)$ belong to the unbinding core. If we assume that the one-hunter team can more smoothly hunt a hare, as the hunting ground is less crowded, and that $V(\{i\})=\{(2+\epsilon)\}, \epsilon>0$, then $C^{\mathrm{UB}}(V)=\{(7,7,7)\}$.
} 


\section{Basic properties of the cores}

\subsection{Coincidence between the cores}

We begin this section by showing some conditions for the unbinding core to coincide with the traditional core. For any $S \subseteq N$ and any $y \in \mathbb{R}^{S}$, we define $D O M^{S}(y, V)=\left\{x \in V(N) \mid y_{j}>x_{j}\right.$ for all $\left.j \in S\right\} \subseteq$ $\mathbb{R}^{N}$. Let $\eta^{S}(V)=\bigcap_{y \in V(S)} D O M^{S}(y, V)$, and similarly, $\mu^{S}(V)=\bigcup_{y \in V(S)} D O M^{S}(y, V)$. We offer the following expressions and conditions. The proofs are provided in the appendix.

\section{Lemma 3.1.}

$$
\begin{aligned}
C(V) & =V(N) \backslash \bigcup_{S \subseteq N} \mu^{S}(V), \\
C^{\mathrm{UB}}(V) & =V(N) \backslash \bigcup_{S \subseteq N} \eta^{S}(V) .
\end{aligned}
$$

Proposition 3.2. The following three statements are equivalent:

$1 C(V)=C^{\mathrm{UB}}(V)$

$2 \bigcup_{S \subseteq N} \mu^{S}(V)=\bigcup_{S \subseteq N} \eta^{S}(V)$

3 for any $x \in V(N), x$ satisfies either

(i) there exists $S \subseteq N$ such that $x \in \eta^{S}(V)$ or

(ii) for any $S \subseteq N, x \notin \mu^{S}(V)$.

Given that $C(V) \subseteq C^{\mathrm{UB}}(V)$, the third condition means that every allocation $x \in V(N)$ either belongs to $C(V)$ or not to $C^{\mathrm{UB}}(V)$, which results in no "gap" between the two cores. Proposition 3.2 shows that the unbinding core coincides with the core if every allocation that is dominated by some allocation through some coalition $S$ is also dominated by all allocations in $V(S)$.

Remark 3.3. The unbinding core satisfies covariance as well as the binding core.

\subsection{Monotonicity}

In the traditional theory of core, an expansion of $V(S)$ implies that coalition $S$ becomes more likely to deviate from $N$ because it offers more options to coalition $S$, which, as a result, reduces the core. However, for the unbinding core, an expansion of $V(S)$ brings about the result opposite to what the following proposition suggests.

Proposition 3.4. Let $V, V^{\prime}$ be games.

$$
V^{\prime}(S) \supseteq V(S) \text { for all } S \subseteq N \Longrightarrow C^{U B}\left(V^{\prime}\right) \supseteq C^{U B}(V)
$$

Proposition 3.4 states that the unbinding core $C^{U B}(V)$ expands to $C^{U B}\left(V^{\prime}\right)$ as $V(S)$ expands to $V^{\prime}(S)$. On the other hand, the formal proposition about the traditional core is provided as follows, which shows that the traditional core shrinks as $V(S)$ expands. 
Proposition 3.5. Let $V, V^{\prime}$ be games.

$$
\begin{aligned}
& V^{\prime}(S) \supseteq V(S) \text { for all } S \subsetneq N \Longrightarrow C\left(V^{\prime}\right) \subseteq C(V) \\
& \text { and } V^{\prime}(N)=V(N)
\end{aligned}
$$

The proofs are provided in the appendix. How can we understand the difference between these propositions? We interpret this result in terms of negotiation and uncertainty. From the negotiation viewpoint, we consider $V(S)$ as the set of allocations that the players in $S$ negotiate with each other to solve their internal conflict. The expansion of $V(S)$ implies that the players of $S$ obtain more options to negotiate, which may make it more difficult to build consensus within $S$ and to deviate from $N$. As for the viewpoint of uncertainty, an expansion of $V(S)$ means that the members of $S$ get more uncertain about which allocation finally results after their deviation. The expansion of the unbinding core exhibits players' cautious attitudes toward the possibility that the worst outcome may become worse.

To examine Propositions 3.4 and 3.5, we consider a symmetric version of Example 2.2: we set $V(\{2,3\})=\{(0.5,0.5)\}$, and the same for the other coalitions. We consider $V^{\prime}$ as the original game in Example 2.2. Clearly, $V^{\prime}(S) \supseteq V(S)$ for all $S \subseteq N$ and $V^{\prime}(N)=V(N)$. Since $|V(S)|=1$ for any $S \subseteq N$, we readily have

$$
C^{\mathrm{UB}}(V)=C(V)=\{(0,0.5,0.5),(0.5,0,0.5),(0.5,0.5,0)\} .
$$

Expanding $V(\{2,3\})$ to $V^{\prime}(\{2,3\})$ shrinks the core to $C\left(V^{\prime}\right)=\{(0,0.5,0.5)\}$ and allows for the unbinding core to contain $L_{a}, L_{b}, A_{a}$, and $A_{b}$, as discussed in Example 2.2.

Now, we focus on "how $V(S)$ expands." The following proposition shows that if $V(S)$ expands to contain modest choices, then it does not affect the unbinding core.

Proposition 3.6. Let $V$ be a game and fix an arbitrary coalition $S^{*} \subsetneq N$. Let $V^{\prime}\left(S^{*}\right)=\operatorname{co}\left(V\left(S^{*}\right)\right)$ and $V^{\prime}(S)=V(S)$ for any $S \subseteq N\left(S \neq S^{*}\right)$. Then, $C^{U B}\left(V^{\prime}\right)=C^{U B}(V)$.

The traditional core does not obey this property. This proposition shows that even if an intermediate choice is added, it does not influence the players' (unbinding) deviation. ${ }^{* 8}$ We use Example 2.2 again to describe Proposition 3.6. Now, let $V$ be the game in Example 2.2. We define $V^{\prime}$ as $V^{\prime}(\{2,3\})=$ $c o(\{(0.4,0.6),(0.6,0.4)\})$ and $V^{\prime}(S)=V(S)$ for any other coalition $S \subseteq N$. It holds that $\eta^{\{2,3\}}\left(V^{\prime}\right)=$ $\eta^{\{2,3\}}(V)$. Hence, $C^{U B}\left(V^{\prime}\right)=C^{U B}(V)$. Note that we can weaken the constraint of Proposition 3.6 by replacing $V^{\prime}\left(S^{*}\right)=\operatorname{co}\left(V\left(S^{*}\right)\right)$ with $V^{\prime}\left(S^{*}\right)=V\left(S^{*}\right) \cup C$, where $C$ is an arbitrary subset of $\operatorname{co}\left(V\left(S^{*}\right)\right)$.

We conclude this subsection with two technical remarks.

Remark 3.7. As for Proposition 3.5, one might hold that $V^{\prime}(N)=V(N)$ is not necessary. Hence, let

\footnotetext{
*8 Note that the following is a corollary of Proposition 3.6: Let $V$ be a game. Let $V^{\prime}(S)=\operatorname{co}(V(S))$ for any $S \subsetneq N$ and $V^{\prime}(N)=V(N)$. Then, $C^{U B}\left(V^{\prime}\right)=C^{U B}(V)$.
} 
us briefly check the role of the condition through Example 2.2. We define $V$ as follows:

$$
\begin{aligned}
V(N) & =\left\{x \in \mathbb{R}^{3} \mid \sum_{j \in N} x_{j}=1, x \neq(0,0.5,0.5)\right\}, \\
V(\{1,2\}) & =\{(0.5,0.5)\} \\
V(\{1,3\}) & =\{(0.5,0.5)\} \\
V(\{2,3\}) & =\{(0.6,0.4),(0.4,0.6)\} \\
V(\{i\}) & =\{(0)\} \text { for all } i \in N .
\end{aligned}
$$

Let $V^{\prime}$ be the game of Example 2.2. Hence, $V^{\prime}(S) \supseteq V(S)$ for all $S \subsetneq N$ and $V^{\prime}(N) \supsetneq V(N)$. However, we have $C(V)=\emptyset \subsetneq\{(0,0.5,0.5)\}=C\left(V^{\prime}\right)$, which violates Proposition 3.5.

Remark 3.8. NTU games (games with nontransferable utility) are often assumed to be comprehensive. A game is comprehensive if for every $S \subseteq N$, if $x \in V(S), y \in \mathbb{R}^{S}$, and $y_{j} \leq x_{j}$ for any $j \in S$, then $y \in V(S)$. Proposition 3.4 suggests that we need to pay attention to comprehensive games: if a game is comprehensive, then no coalition unbindingly deviates, and the unbinding core is $V(N)$. This is because of the pessimism of the unbinding core: the comprehensiveness of $V(S)$ is considered the possibility that a boundlessly bad allocation may result in an outcome of $S$ 's deviation. This contrasts with the optimistic attitude that the traditional definition of deviation describes. Fortunately, by slightly changing the definition of unbinding deviation, we can avoid this technical problem and apply the spirit of unbinding deviation to comprehensive games. Let $V$ be an arbitrary game. We now define $S \operatorname{dev}^{*} \mathrm{UB} x$ as, for all $y \in V^{*}(S), y_{j}>x_{j}$ for any $j \in S$, where $V^{*}(S)$ is the set of efficient allocations given by $V^{*}(S)=\left\{y \in V(S) \mid \nexists y^{\prime} \in V(S)\right.$ s.t. $y_{j}^{\prime} \geq y_{j}$ for all $j \in S, y_{k}^{\prime}>y_{k}$ for some $\left.k \in S\right\}$. Let $C^{*} \mathrm{UB}(V)=$ $\left\{x \in V^{*}(N) \mid \nexists S \subseteq N\right.$ s.t. $\left.S \operatorname{dev}^{*}{ }^{*} x\right\}$. It readily follows that $C^{*} \mathrm{UB}(V)=C^{\mathrm{UB}}\left(V^{*}\right)$.

\subsection{The relationship with TU games}

In the previous subsection, we compared the unbinding core with the traditional core. In this subsection, we study the relationship with games with transferable utility (TU games) by constructing $V$ from a TU game $v$. Let $v$ be a TU game, i.e., $v: 2^{N} \rightarrow \mathbb{R}$. The core of a TU game $v$ is the set of allocations from which no coalition deviates. A coalition $S$ deviates from allocation $x(S \operatorname{dev} x)$ if there exists $y \in \mathbb{R}^{S}$ such that $\sum_{j \in S} y_{j} \leq v(S)$ and $y_{j}>x_{j}$ for any $j \in S$. Therefore, the core of a TU game is formally defined as follows:

$$
C(v)=\left\{x \in \mathbb{R}^{N} \mid \sum_{j \in N} x_{j}=v(N) \text { and } \nexists S \subseteq N \text { such that } S \operatorname{dev} x\right\} .
$$

Similarly, we define the set of imputations as follows:

$$
\begin{aligned}
I(v) & =\left\{x \in \mathbb{R}^{N} \mid \sum_{j \in N} x_{j}=v(N) \text { and } x_{i} \geq v(\{i\}) \text { for all } i \in N\right\} \\
& =\left\{x \in \mathbb{R}^{N} \mid \sum_{j \in N} x_{j}=v(N) \text { and } \nexists i \in N \text { such that } i \operatorname{dev} x\right\} .
\end{aligned}
$$


First, we examine the most standard construction of $V(S)$ : the set of allocations whose sum is equal to $v(S)$. For any TU game $v$, we define $V_{v}(S)=\left\{x \in \mathbb{R}^{S} \mid \sum_{j \in S} x_{j}=v(S)\right\}$ for any $S \subseteq N$.

Proposition 3.9. For any TU game $v$,

$$
C(v)=C\left(V_{v}\right) \subseteq C^{U B}\left(V_{v}\right)=I(v) .
$$

The proof is provided in the appendix. The reason behind this result is similar to Remark 3.8: some allocation that is not profitable for some members in $S$ prevents $S$ from deviating. In other words, as long as $V(S)$ is not bounded, similar results hold. Next, we consider restricting $V(S)$ to a particular value. For any $S \subseteq N$, we define

$$
V_{v}^{\mathrm{ED}}(S)=\left(\frac{v(S)}{|S|}, \ldots, \frac{v(S)}{|S|}\right) \in \mathbb{R}^{S} .
$$

Clearly, $\left|V_{v}^{\mathrm{ED}}(S)\right|=1$ for any $S \subseteq N$. Therefore, we have $C^{U B}\left(V_{v}^{\mathrm{ED}}\right)=C\left(V_{v}^{\mathrm{ED}}\right)$ for any TU game $v$. We denote it by $C^{E D}(v)$. The following result is not difficult to check.

\section{Proposition 3.10.}

$$
C^{\mathrm{ED}}(v) \neq \emptyset \Longleftrightarrow \text { for any } S \subseteq N, \frac{v(S)}{|S|} \leq \frac{v(N)}{|N|} .
$$

The proof is provided in the appendix. Proposition 3.10 and $C^{\mathrm{ED}}$ describe the stability of the equal division of $v(N)$ by assuming that members of every deviating coalition share its profit $v(S)$ equally. Selten $(1972,1987)$ studied a similar concept, called the equal division core. The only difference is that the equal division core does not restrict the allocations for $v(N)$ into the equal division: setting $V_{v}^{\mathrm{ED} *}(S)=V_{v}^{\mathrm{ED}}(S)$ for every $S \subsetneq N$ and $V_{v}^{\mathrm{ED} *}(N)=\left\{x \in R^{N} \mid \sum_{j \in N} x_{j}=v(N)\right\}$, we define the equal division core as $C^{E D *}(v)=C^{U B}\left(V_{v}^{\mathrm{ED} *}\right)=C\left(V_{v}^{\mathrm{ED} *}\right){ }^{* 9}$ He investigated the equal division core through experiments to observe subjects' behavioral equity. On the other hand, Bhattacharya (2004) offers a theoretical justification for the equal division core. He proposes some axioms including antimonotonicity as Proposition 3.5 and provides an axiomatic characterization.

The equal division is not the only way to divide the surplus. We now consider the Shapley value instead of the equal division. In the same manner as $C^{\mathrm{ED}}(v)$ and $C^{\mathrm{ED} *}(v)$, we define $C^{\mathrm{Sh}}(v)$ and $C^{\mathrm{Sh} *}(v)$. In other words, we assume that deviating players share the profit $v(S)$ by the Shapley value of the subgame $\left.v\right|_{S}$ after their deviation. It may be clear that balanced games are sufficient for $C^{\mathrm{Sh} *}(v)$ to be nonempty. However, this is not true for $C^{\mathrm{Sh}}(v)$. An example is a gloves game:

$$
v(N)=v(\{1,2\})=v(\{1,3\})=1 \text { and } v(\{2,3\})=v(\{i\})=0 .
$$

The Shapley value for the grand coalition is $\left(\frac{2}{3}, \frac{1}{6}, \frac{1}{6}\right)$, whereas for $\{1,2\}$ and $\{1,3\}$, the Shapley value is $\left(\frac{1}{2}, \frac{1}{2}\right)$. Hence, player 1 does not agree with the deviations $\{1,2\}$ and $\{1,3\}$.

We can employ any solution concept $\phi$ as the way to distribute $v(S)$ and define $C^{\phi}(v)$ and $C^{\phi *}(v)$ in the same manner. Below, we conclude this section by some brief corollaries and a remark derived from the above observation.

*9 Selten originally defined the equal division core in more general settings. He did not necessarily assume that allocations in the equal division core are efficient for the grand coalition. 
- If $\phi$ is a set-valued solution, then $C\left(V_{v}^{\phi}\right)$ may not coincide with $C^{U B}\left(V_{v}^{\phi}\right)$.

- For any $\phi$, we have $C^{U B}\left(V_{v}^{\phi *}\right) \supseteq C\left(V_{v}^{\phi *}\right) \supseteq C(v)$. Hence, balanced games are sufficient for them to be nonempty.

- For any $\phi$, if $\phi(v) \subseteq C(v)$ (or $\phi(v) \in C(v)$ ), then $C^{U B}\left(V_{v}^{\phi}\right)$ and $C\left(V_{v}^{\phi}\right)$ are nonempty.

Remark 3.11. For any single-valued solution $\phi$, vector $\left(\phi\left(\left.v\right|_{S}\right)\right)_{S \subseteq N}$ can be seen as an allocation scheme of $v$, namely, a vector assigning an allocation $x^{S} \in \mathbb{R}^{S}$ to every $S \subseteq N$. Sprumont (1990) defines population monotonicity for allocation schemes: for any $S, T$ with $S \subseteq T, x_{j}^{S} \leq x_{j}^{T}$ for any $j \in S$. We can think of $C^{\phi}(v)$ as another stability for allocation schemes because it requires $\phi_{j}\left(\left.v\right|_{S}\right) \leq \phi_{j}(v)$ for any $S \subseteq N$ and any $j \in S$. This stability is weaker than population monotonicity. Population monotonicity guarantees a process of coalition formation to the grand coalition, while our concept prevents the grand coalition from breaking up into partial coalitions.

\section{The balancedness of the unbinding core}

In this section, we provide a general sufficient condition for the unbinding core to be nonempty. This is an extension of Shapley and Vohra (1991)'s approach using Kakutani's fixed point theorem. ${ }^{* 10}$

Let $V^{+}(S)=\left\{x^{\prime} \in \mathbb{R}^{N} \mid \exists x \in V(S)\right.$ such that $\left.x_{j}^{\prime} \leq x_{j} \forall j \in S\right\}$. Define $\widehat{D O M}^{S}(y, V)=\left\{x \in \mathbb{R}^{N} \mid y_{j}>\right.$ $x_{j}$ for all $\left.j \in S\right\}$ and $\hat{\eta}^{S}(V)=$ the closure of $\bigcap_{y \in V(S)} \widehat{D O M}^{S}(y, V)$. Throughout this section, we assume that $|V(\{i\})|=1$ and $V(\{i\}) \in \mathbb{R}_{++}$for any $i \in N$. Define $e^{S} \in \mathbb{R}^{N}$ as $e_{i}^{S}=1$ if $i \in S$ and 0 if $i \notin S$. A collection of coalitions, $\mathcal{B}$, is balanced if there exist nonnegative weights $\lambda^{S} \in \mathbb{R}_{+}, S \in \mathcal{B}$, such that

$$
\sum_{S \in \mathcal{B}} \lambda^{S} e^{S}=e^{N}
$$

Definition 4.1. A game $V$ is unbindingly balanced if for any balanced collection $\mathcal{B}$,

$$
\bigcap_{S \in \mathcal{B}} \hat{\eta}^{S}(V) \subseteq V^{+}(N)
$$

Proposition 4.2. If a game $V$ is unbindingly balanced, then $C^{\mathrm{UB}}(V) \neq \emptyset$.

The proof is provided in the appendix. The game in Example 2.1, namely, the stag hunt game, is unbindingly balanced. In this paper, our "game" does not necessarily mean an NTU game, which is usually supposed to satisfy comprehensiveness, convexity, and closedness. ${ }^{* 11}$ In the proof, we extend Shapley and Vohra's (1991) approach to our settings. We can also extend the condition for the traditional core to our framework. The extended condition for the traditional core is weaker than the condition above.

\footnotetext{
*10 For a constructive proof of the core for NTU games, see Scarf (1967). A necessary and sufficient condition is provided by Predtetchinski and Herings (2004).

${ }^{* 11}$ If $V(S)$ is closed for every $S \subseteq N$, we can use $\eta^{S}(V)$ instead of its closure $\hat{\eta}^{S}(V)$.
} 


\section{Credible coalitions}

Ray (1989) introduces the notion of credible coalition and proposes a modified core concept called the credible core. He shows that the traditional core and the credible (traditional) core are identical. His investigation is also motivated by a similar concern: Clearly, coalitions incapable of reaching an agreement within its members are not "credible" (Ray 1989, p. 186). In this section, we extend his notion to the unbinding core. We begin with the definition of credible coalition.

Definition 5.1. A coalition $S$ is credible if for some $x \in V(S)$, there is no credible coalition $T \subsetneq S$ such that $T \operatorname{dev} x$. Every one-person coalition is credible.

Let $C C(V)$ be the credible core of game $V$, namely, the set of allocations from which no credible coalition deviate. Clearly, $C(V) \subseteq C C(V)$ for any game $V$. Ray (1989) shows that $C(V)=C C(V)$ for any game $V$. Now, how do we describe credibility for unbinding deviations? We introduce the following two plausible forms of credibility for unbinding deviation.

\section{Definition 5.2.}

- A coalition $S$ is credible $e^{U B}$ if for some $x \in V(S)$, there is no credible ${ }^{\mathrm{UB}}$ coalition $T \subsetneq S$ such that $T$ dev $^{\mathrm{UB}} x$. Every one-person coalition is credible ${ }^{\mathrm{UB}}$.

- A coalition $S$ is consistently credible $e^{U B}\left(\right.$ credible $\left.^{* U B}\right)$ if for all $x \in V(S)$, there is no credible*UB coalition $T \subsetneq S$ such that $T \operatorname{dev}^{\mathrm{UB}} x$. Every one-person coalition is credible*UB .

The former is a simple extension of Definition 5.1, where we replace dev with $\mathrm{dev}^{\mathrm{UB}}$. In other words, for some $x \in V(S)$ and for any proper subcoalition $T$ of $S, T$ does not unbindingly deviate from $x$. On the other hand, the latter requires that for any $x \in V(S)$ and any proper subcoalition $T$ of $S, T$ does not unbindingly deviate from $x$. Therefore, the latter version of credibility may more consistently exhibit the spirit of the unbinding deviation. We define $C C^{\mathrm{UB}}(V)$ and $C C^{* \mathrm{UB}}(V)$ in the same manner:

$$
\begin{aligned}
C C^{\mathrm{UB}}(V) & =\left\{x \in V(N) \mid \nexists \text { credible }^{\mathrm{UB}} S \subset N \text { such that } S \mathrm{dev}^{\mathrm{UB}} x\right\}, \\
C C^{* \mathrm{UB}}(V) & =\left\{x \in V(N) \mid \nexists \text { credible }^{* \mathrm{UB}} S \subset N \text { such that } S \operatorname{dev}^{\mathrm{UB}} x\right\} .
\end{aligned}
$$

It follows that $C^{\mathrm{UB}}(V) \subseteq C C^{\mathrm{UB}}(V)$ and $C^{\mathrm{UB}}(V) \subseteq C C^{* \mathrm{UB}}(V)$ for any $V$. The following result shows that $C^{\mathrm{UB}}, C C^{\mathrm{UB}}(V)$, and even $C C^{* \mathrm{UB}}(V)$ are identical. The proof is provided in the appendix.

Proposition 5.3. For any game $V, C^{\mathrm{UB}}(V)=C C^{\mathrm{UB}}(V)=C C^{* \mathrm{UB}}(V)$.

Given the consistent recursion of credibility $^{* \mathrm{UB}}$, credibility $^{\mathrm{UB}}$ should be compatible with binding deviation. We associate unbindingness with credibility ${ }^{* \mathrm{UB}}$ and bindingness with credibility ${ }^{\mathrm{UB}}$. Proposition 5.3 shows that whether bindingness or unbindingness, whichever supports each deviating coalition's credibility, the unbinding core is identical. The traditional core does not obey this property. To see this, we define credible* $^{*}$ in the same manner as Definition 5.2.

Definition 5.4. A coalition $S$ is credible* if for all $x \in V(S)$, there is no credible* coalition $T \subsetneq S$ such 
that $T \operatorname{dev} x$. Every one-person coalition is credible*.

We use $C C^{*}(V)$ to denote the credible core based on Definition 5.4. It is worth mentioning that the two credibility concepts, Definitions 5.1 and 5.4 , are independent as follows. Let $N=\{1,2,3\}$,

$$
\begin{aligned}
V(N) & =\left\{x \in \mathbb{R}^{3} \mid \sum_{j \in N} x_{j}=1\right\}, \\
V(\{i, j\}) & =\{(0.4,0.4),(0.7,0.1),(0.1,0.7)\} \text { for all } i, j \in N(i \neq j), \\
V(\{i\}) & =\{(0.2)\} \text { for all } i \in N .
\end{aligned}
$$

In this example, two-person coalitions are credible but not credible* . Moreover, we have $C C \subsetneq C C^{*}$; for example, $(0.6,0.2,0.2) \in C C^{*} \backslash C C$. Now, let $N=\{1,2,3,4\}$.

$$
\begin{aligned}
V(N) & =\left\{x \in \mathbb{R}^{4} \mid \sum_{j \in N} x_{j}=1\right\}, \\
V(\{i, j, k\}) & =\{(0.3,0.3,0.3)\} \text { for all } i, j, k \in N(i \neq j \neq k \neq i), \\
V(\{i, j\}) & =\{(0.4,0.4),(0.7,0.1),(0.1,0.7)\} \text { for all } i, j \in N(i \neq j), \\
V(\{i\}) & =\{(0.2)\} \text { for all } i \in N .
\end{aligned}
$$

In this example, three-person coalitions are credible* but not credible. Similarly, the unbinding versions of credibility in Definition 5.2 are mutually independent.

\section{Concluding remarks}

We conclude this paper with remarks on weak deviation and future work. In this paper, we employ the strict form of deviation: $y_{j}>x_{j}$ for any $j \in S$. In many preceding works (in particular, TU games), weak deviation is also studied. A coalition $S$ weakly deviates from an allocation $x \in \mathbb{R}^{N}$ if there exists $y \in V(S)$ such that $y_{j} \geq x_{j}$ for any $j \in S$ and $y_{i}>x_{i}$ for some $i \in S$. This change similarly applies to the unbinding deviation, $D O M, \eta$, and $\mu$. The results provided in this paper hold even with weak deviation. Below is a brief technical remark for the balancedness.

As defined in Section 4, as long as we employ strict deviation, $\widehat{D O M}^{S}(y, V)$ is a open set. In contrast, if we employ weak deviation, $\widehat{D O M}^{S}(y, V)$ is a closed set whenever $V(S)$ is not a singleton. If $V(S)$ consists of a single vector, then $\widehat{D O M}^{S}(y, V)$ is neither open nor closed. This needs a slight modification. If $V(S)$ is a singleton for any $S \subsetneq N$, then we can replace the definition of $\hat{\eta}^{S}(V)$ with the following form:

$$
\hat{\eta}^{S}(V)=\bigcap_{y \in V(S)} \widehat{D O M}^{S}(y, V),
$$

where we note that the intersection of (finite/ infinite number of) closed sets is closed. Proposition 4.2 remains true by this modification.

The notion of an unbinding core can be a useful approach to the problems that have empty (traditional) core and discrete allocations - for example, Shapley-Scarf's trading economy with multiple types of indivisible goods. Shapley and Scarf's (1974) well-known result is that the core is nonempty as long as the number of types of indivisible goods, say, $Q$, is 1 . The same question in the case of $Q=2$ is brought 
up by Moulin (1995). Konishi et al. (2001) answer this question and show that the core may be empty if $Q \geq 2$. Precisely, even if players' preferences are strict and additively separable, the nonemptiness of the core is not necessarily guaranteed. ${ }^{* 12}$ As discussed through this paper, the unbinding core becomes a superset of the traditional core. We conjecture that the unbinding core can be nonempty even for $Q \geq 2$.

In addition, the unbinding core may be helpful for analyzing the vNM stable set (von Neumann and Morgenstern 1944). As briefly mentioned in Subsection 3.3, the equal division core $C^{E D *}$ can be thought of as a specific form of unbinding core and coincides with the vNM stable set for some simple games; e.g., for the three-person symmetric majority game, they are $\{(0.5,0.5,0),(0.5,0,0.5),(0,0.5,0.5)\}$. Considering the difficulty of analyzing the vNM stable set, "approximating" the vNM stable set by the unbinding core might become a good step for studying the vNM stable set.

\section{Appendix}

\section{Proof of Lemma 3.1}

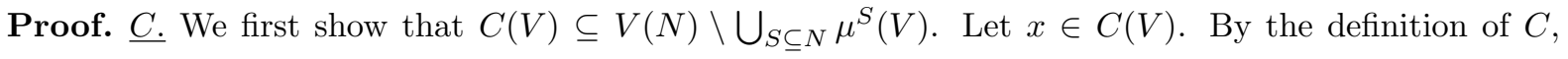
there is no $S$ such that $S \operatorname{dev} x$. Hence, there is no $S$ such that for some $y \in V(S), y_{j}>x_{j}$ for any $j \in S$, or equivalently, there is no $S$ such that for some $y \in V(S), x \in D O M^{S}(y, V)$. In other words, for any $S$, we have $x \notin D O M^{S}(y, V)$ for any $y \in V(S)$. Hence, for every $S \subseteq N, x \notin \mu^{S}(V)$. We obtain $x \notin \bigcup_{S \subseteq N} \mu^{S}(V)$. The opposite inclusion relation holds by proceeding from bottom to top.

$C^{\mathrm{UB}}$. Now, we show that $C^{\mathrm{UB}}(V) \subseteq V(N) \backslash \bigcup_{S \subseteq N} \eta^{S}(V)$. Let $x \in C^{\mathrm{UB}}(V)$. By the definition of $C^{\mathrm{UB}}$, there is no $S$ such that $S \operatorname{dev}^{\mathrm{UB}} x$. Hence, there is no $S$ such that for all $y \in V(S), y_{j}>x_{j}$ for any $j \in S$, or equivalently, there is no $S$ such that for all $y \in V(S), x \in D O M^{S}(y, V)$. In other words, for any $S$, there exists $y \in V(S)$ such that $x \notin D O M^{S}(y, V)$. Hence, for every $S \subseteq N, x \notin \eta^{S}(V)$. We obtain $x \notin \bigcup_{S \subseteq N} \eta^{S}(V)$. The opposite inclusion relation holds in the same manner.

\section{Proof of Proposition 3.2}

Proof. It is clear from Lemma 3.1 that 1 is equivalent to 2 . We show that 1 is equivalent to 3 .

$(\Longleftarrow)$ We show that $C(V) \supseteq C^{\mathrm{UB}}(V)$. Let $x \in C^{\mathrm{UB}}(V)$. The allocation $x$ must satisfy either (i) or (ii). If $x$ satisfies (i), it follows from Lemma 3.1 that $x \notin C^{\mathrm{UB}}(V)$, which contradicts the assumption $x \in C^{\mathrm{UB}}(V)$. Hence, the allocation $x$ satisfies (ii), namely, $x \notin \bigcup_{S \subseteq N} \mu^{S}(V)$. By Lemma 3.1, $x \in C(V)$.

$(\Longrightarrow)$ Assuming that there exists $x \in V(N)$ satisfying neither (i) nor (ii), we show that $x \in C^{\mathrm{UB}}(V) \backslash$ $C(V)$. From the assumption, it follows that [for any $S, x \notin \eta^{S}(V)$ ] and [there exists $S$ such that $\left.x \in \mu^{S}(V)\right]$. By Lemma 3.1, the former implies $x \in C^{\mathrm{UB}}(V)$, and the latter $x \notin C(V)$. This completes the proof.

*12 To be even more precise, the core is nonempty if $(n=3$ and $Q=2), n \leq 2$, or $Q=1$. 


\section{Proof of Proposition 3.4}

Proof. Let $x \in C^{U B}(V)$. From the definition of $C^{U B}$, it follows that

$$
C^{U B}(V)=\left\{x^{\prime} \in V(N) \mid \nexists S \subseteq N \text { s.t. for any } y \in V(S) \text { and any } j \in S, y_{j}>x_{j}^{\prime}\right\} \text {. }
$$

Hence, $x$ satisfies (i) and there is no $S \subseteq N$ such that for any $y \in V(S)$ and any $j \in S, y_{j}>x_{j}$, and (ii) $x \in V(N)$. Condition (i) is the same as the following statement:

for all $S \subseteq N$, there exist $y \in V(S)$ and $j \in S$ such that $y_{j} \leq x_{j}$.

Hence, from the assumption $V^{\prime}(S) \supseteq V(S)$ for all $S \subseteq N$, we obtain (i')

For all $S \subseteq N$, there exist $y \in V^{\prime}(S)$ and $j \in S$ such that $y_{j} \leq x_{j}$.

and (ii') $x \in V^{\prime}(N)$. Condition (i') means that there is no $S \subseteq N$ such that for any $y \in V^{\prime}(S)$ and any $j \in S, y_{j}>x_{j}$. Thus, we obtain

$$
x \in\left\{x^{\prime} \in V^{\prime}(N) \mid \nexists S \subseteq N \text { s.t. for any } y \in V^{\prime}(S) \text { and any } j \in S, y_{j}>x_{j}^{\prime}\right\}=C^{U B}\left(V^{\prime}\right) .
$$

\section{Proof of Proposition 3.5}

Proof. The proof is an analog of Proposition 3.4. Let $x \in C\left(V^{\prime}\right)$. From the definition of $C$, it follows that

$$
C\left(V^{\prime}\right)=\left\{x^{\prime} \in V^{\prime}(N) \mid \nexists S \subseteq N \text { s.t. for some } y \in V^{\prime}(S) \text { and any } j \in S, y_{j}>x_{j}^{\prime}\right\}
$$

Hence, $x$ satisfies (i) there is no $S \subseteq N$ such that for some $y \in V^{\prime}(S)$ and any $j \in S, y_{j}>x_{j}$, and (ii) $x \in V^{\prime}(N)$. Condition (i) is the same as the following statement:

for all $S \subseteq N$ and any $y \in V^{\prime}(S)$, there exists $j \in S$ such that $y_{j} \leq x_{j}$.

Hence, from the assumptions $V^{\prime}(S) \supseteq V(S)$ for all $S \subsetneq N$ and $V^{\prime}(N)=V(N)$, we obtain (i')

For all $S \subseteq N$ and any $y \in V(S)$, there exists $j \in S$ such that $y_{j} \leq x_{j}$.

and (ii') $x \in V^{\prime}(N)$. Condition (i') means that there is no $S \subseteq N$ such that for some $y \in V(S)$ and any $j \in S, y_{j}>x_{j}$. Thus, we obtain

$x \in\left\{x^{\prime} \in V(N) \mid \nexists S \subseteq N\right.$ s.t. for some $y \in V(S)$ and any $\left.j \in S, y_{j}>x_{j}^{\prime}\right\}=C(V)$. 


\section{Proof of Proposition 3.6}

Proof. We fix $S \subsetneq N$ (this fixed $S$ is the $S^{*}$ in the statement of Proposition 3.6. We omit $*$ in this proof for simplicity). From Lemma 3.1, it suffices to show that $\eta^{S}(V)=\eta^{S}\left(V^{\prime}\right)$.

$\underline{\eta^{S}(V) \supseteq \eta^{S}\left(V^{\prime}\right)}$. We have $V^{\prime}(S)=c o(V(S)) \supseteq V(S)$. Hence, we have

$$
\begin{aligned}
& \eta^{S}(V)=\bigcap_{y \in V(S)} \operatorname{DOM}^{S}(y, V) \\
&=\bigcap_{y \in V(S)}\left\{x \in V(N) \mid y_{j}>x_{j} \forall j \in S\right\} \\
& V^{\prime}(S) \supseteq V(S) \bigcap_{y \in V^{\prime}(S)}\left\{x \in V(N) \mid y_{j}>x_{j} \forall j \in S\right\} \\
& \stackrel{\bigcap^{\prime}(N)=V(N)}{=} \bigcap_{y \in V^{\prime}(S)}\left\{x \in V^{\prime}(N) \mid y_{j}>x_{j} \forall j \in S\right\} \\
&=\bigcap_{y \in V^{\prime}(S)} D O M^{S}\left(y, V^{\prime}\right) \\
&= \\
& \eta^{S}\left(V^{\prime}\right) .
\end{aligned}
$$

$\eta^{S}(V) \subseteq \eta^{S}\left(V^{\prime}\right)$. Suppose that there exists $x$ such that $x \in \eta^{S}(V)$ and $x \notin \eta^{S}\left(V^{\prime}\right)$. First, it follows from $x \in \eta^{S}(V)$ that for any $y \in V(S), x \in D O M^{S}(y, V)$. Hence, we have

$$
\text { for any } y \in V(S) \text { and any } j \in S, x_{j}<y_{j} \text {. }
$$

Next, $x \notin \eta^{S}\left(V^{\prime}\right)$ implies that $x \notin \bigcap_{y \in V^{\prime}(S)} D O M^{S}\left(y, V^{\prime}\right)$. Hence, there exists $y^{*} \in V^{\prime}(S)$ such that $x \notin D O M^{S}\left(y^{*}, V^{\prime}\right)$, equivalently, in view of $V^{\prime}(N)=V(N)$,

$$
\text { there exists } y^{*} \in V^{\prime}(S) \text { such that } x \notin\left\{x^{\prime} \in V(N) \mid y_{j}^{*}>x_{j}^{\prime} \forall j \in S\right\} \text {. }
$$

Since $y^{*} \in V^{\prime}(s)$ and $V^{\prime}(S)=c o(V(S))$, there exist $y^{1}, \ldots, y^{m} \in V(S)$ and coefficients $a^{1}, \ldots, a^{m}$ such that $a^{1}+\ldots+a^{m}=1,0 \leq a^{l} \leq 1$ for every $1 \leq l \leq m$, and

$$
y_{j}^{*}=a^{1} y_{j}^{1}+\ldots+a^{m} y_{j}^{m} \text { for any } j \in S .
$$

Moreover, it follows from (A.2) that there exists $j^{*} \in S$ such that $x_{j^{*}} \geq y_{j^{*}}^{*}$. By (A.3), we have

$$
x_{j^{*}} \geq a^{1} y_{j^{*}}^{1}+\ldots+a^{m} y_{j^{*}}^{m} .
$$

In view of (A.1), we have

$$
\begin{aligned}
& y_{j^{*}}^{1}>a^{1} y_{j^{*}}^{1}+\ldots+a^{m} y_{j^{*}}^{m}, \\
& y_{j^{*}}^{2}>a^{1} y_{j^{*}}^{1}+\ldots+a^{m} y_{j^{*}}^{m}, \\
& \quad \cdots \\
& y_{j^{*}}^{m}>a^{1} y_{j^{*}}^{1}+\ldots+a^{m} y_{j^{*}}^{m},
\end{aligned}
$$

which implies

$$
\begin{aligned}
a^{1} y_{j^{*}}^{1}+\ldots+a^{m} y_{j^{*}}^{m} & >\left(a^{1}+\ldots+a^{m}\right)\left(a^{1} y_{j^{*}}^{1}+\ldots+a^{m} y_{j^{*}}^{m}\right) \\
& =\left(a^{1} y_{j^{*}}^{1}+\ldots+a^{m} y_{j^{*}}^{m}\right) .
\end{aligned}
$$


This is a contradiction.

\section{Proof of Proposition 3.9}

Proof. $C(v) \subseteq C\left(V_{v}\right)$. Let $x \in C(v)$. There is no $(S, y)$ satisfying $y_{j}>x_{j}$ for any $j \in S$ and $\sum_{j \in S} y_{j} \leq$ $v(S)$. Hence, there is no $(S, y)$ satisfying $y_{j}>x_{j}$ for any $j \in S$ and $\sum_{j \in S} y_{j}=v(S)$. By the definition of $V_{v}(S)$, no $(S, y)$ satisfies $y_{j}>x_{j}$ for any $j \in S$ and $y \in V_{v}(S)$, which implies $x \in C\left(V_{v}\right)$.

$C(v) \supseteq C\left(V_{v}\right)$. Let $x \in C(v)$. Similar to the above, there is no $(S, y)$ satisfying $y_{j}>x_{j}$ for any $j \in S$ and $\sum_{j \in S} y_{j}=v(S)$. Assume that some $y^{*}$ satisfies $y_{j}^{*}>x_{j}$ for any $j \in S$ and $\sum_{j \in S} y_{j}^{*}<v(S)$. Then, we define $z^{*}$ as $z_{j}^{*}=y_{j}^{*}+\frac{v(S)-\sum_{j \in S} y_{j}^{*}}{|S|}$ and obtain $\sum_{j \in S} x_{j}^{*}=v(S)$. This is a contradiction. Thus, $x \in C(v)$.

$C^{U B}\left(V_{v}\right) \subseteq I(v)$. Let $x \in C\left(V_{v}\right)$. No coalition $S$ unbindingly deviates from $x$. Hence, for any $S \subseteq N$, there exists $y \in V_{v}(S)$ such that $y_{j} \leq x_{j}$ for some $j \in S$. Thus, for any $i \in N$, there exists $y_{i} \in V_{v}(\{i\})$ such that $y_{i} \leq x_{i}$, or equivalently, $v(\{i\})=y_{i} \leq x_{i}$ for every $i \in N$.

$C^{U B}\left(V_{v}\right) \supseteq I(v)$. Let $x \in I(v)$. We have $x_{i} \geq v(\{i\})$ for any $i \in N$. Hence, for any $S \subseteq N$, if $|S|=1$, then $S$ does not unbindingly deviate from $x$. If $|S| \geq 2, V_{v}(S)$ is given by $V_{v}(S)=\left\{x^{\prime} \in \mathbb{R}^{S} \mid \sum_{j \in S} x_{j}^{\prime}=\right.$ $v(S)\}$. Now, for any $S(|S| \geq 2)$, we fix $s^{*} \in S$ and define $y^{S} \in \mathbb{R}^{S}$ as $y_{s^{*}}^{S}=x_{s^{*}}$ and $y_{j}^{S}=\frac{v(S)-y_{s^{*}}}{|S|-1}$ for any $j \in S \backslash\left\{s^{*}\right\}$. Since $\sum_{j \in S} y_{j}^{S}=v(S)$, we have $y^{S} \in V_{v}(S)$. In other words, for any $S(|S| \geq 2)$, there exists $y^{S} \in V_{v}(S)$ such that $y_{s^{*}}^{S}=x_{s^{*}}$. This implies that no coalition $S(|S| \geq 2)$ unbindingly deviates from $x$.

\section{Proof of Proposition 3.10}

Proof. No coalition deviates from $N$ (in the sense of equal division) if and only if

$$
\text { for any } S \subseteq N \text {, there exists } j \in S \text { such that } \frac{v(S)}{|S|} \leq \frac{v(N)}{|N|} \text {. }
$$

The inequality does not depend on any player in $S$ because the allocation is the equal division of $v(S)$. Hence, the statement above holds if and only if

$$
\text { for any } S \subseteq N, \frac{v(S)}{|S|} \leq \frac{v(N)}{|N|} \text {. }
$$

\section{Proof of Proposition 4.2}

Proof. For any coalition $S$, let $x^{S} \in \mathbb{R}^{S}$ be a restriction of $x$ on $S$, namely, $x_{i}^{S}=x_{i}$ for every $i \in S$. For notational convenience, we write $x \geq y(x>y)$ to denote $x_{i} \geq y_{i}\left(x_{i}>y_{i}\right)$ for any $i \in N$.

Let $V$ be an unbindingly balanced game. We define $Q$ and $W$ as follows. Let $Q=\left\{x \in \mathbb{R}^{N} \mid x \leq q e^{N}\right\}$, where $q$ is a positive real number satisfying for any $S \in 2^{N}$,

$$
x^{S} \in \hat{\eta}^{S}(V) \text { and } x^{S} \geq 0 \Longrightarrow x_{j}<q \text { for any } j \in S .
$$


We define $W$ as

$$
W=\left(\bigcup_{S \subseteq N} \hat{\eta}^{S}(V)\right) \cap Q
$$

Note that $W$ is also comprehensive because $\hat{\eta}^{S}(V)$ and $Q$ are comprehensive.

The set $W$ has the following two properties:

i. $u \in \partial W$ and $u^{\prime}>u \Rightarrow u^{\prime} \notin \partial W$,

ii. $u \in \partial W$ and $u_{j}=0$ for some $j \in N \Rightarrow u_{i}=q$ for some $i \in N$,

where $\partial W$ is the boundary of $W$. The second property follows from the assumption $V(\{i\}) \in \mathbb{R}_{++}$for any $i \in N$.

Let $A$ be a standard simplex: $A=\left\{x \in \mathbb{R}_{+}^{N} \mid \sum_{j \in N} x_{j}=1\right\}$. We now define

$$
f(x)=\{y \in \partial W \mid y=t x \text { for some } t \geq 0\} \text { for any } x \in A \text {. }
$$

Claim 1. $f$ is nonempty, single-valued, and continuous.

Proof. We first show that $f(x) \neq \emptyset$ for any $x \in A$. From (A.5), $q>0$, and $V(\{i\}) \in \mathbb{R}_{++}$for any $i \in N$, it follows that $0 \in W$. Moreover, $W \subseteq Q$ implies that $n(q+1) x \notin W$ for any $x \in A$. Hence, for any $x \in A$, there exists $t \in[0, n(q+1)]$ such that $t x \in \partial W$.

Next, we show that $f$ is single-valued. Assume that $f$ is not single-valued. Then, for some $x \in A$, there exist $y, \bar{y} \in f(x)(y \neq \bar{y})$ such that $y=t x$ and $\bar{y}=\bar{t} x$. Hence, $t \neq \bar{t}$ and let $\bar{t}>t$. We consider two cases. Case 1: $x_{i}>0$ for any $i \in N$. Then, we have $\bar{y}_{i}=\bar{t} x_{i}>t x_{i}=y_{i}$ for any $i \in N$. However, (i) of $W$ 's properties contradicts $\bar{y} \in f(x) \subseteq \partial W$. Case 2: $x_{i}=0$ for some $i \in N$. Define $K=\left\{k \in N \mid x_{k}=0\right\}$ and $I=\left\{i \in N \mid x_{i}>0\right\}$. For any $i \in I$, we have $t x_{i}<\bar{t} x_{i} \leq q$, where $\leq$ follows from $\bar{t} x \in f(x) \subseteq \partial W \subseteq Q$. For any $k \in K, t x_{k}=0$. However, this contradicts (ii) of $W$ 's properties.

Finally, we show that $f$ is continuous. To see this, we show that $f(A)=\{f(x) \mid x \in A\} \partial W$ is closed. Let $y^{k} \rightarrow y^{*}\left(y^{k} \in f(A)\right.$ for any $\left.k\right)$. Assume that $y^{*} \notin f(A)$. Then, we have two cases: Case $1: y^{*} \notin \partial W$ or Case 2: $y^{*} \neq t x$ for any $x \in A$ and any $t \geq 0$. We begin with Case 1. If $y^{*} \notin \partial W$, then $y^{*}$ lies in the interior or exterior of $W$. We assume that $y^{*}$ is an interior point of $W$. We denote the interior of $W$ by $\operatorname{int} W$. There exists an open set $U^{*} \subseteq \operatorname{int} W$ such that $y^{*} \in U^{*}$. Since $y^{*}$ is the point to which the sequence $y^{k}$ converges, for the open set $U^{*}$, there exists $k^{*}$ such that $y^{k^{*}} \in U^{*}$. By $U^{*} \subseteq \operatorname{int} W$, we have $y^{k^{*}} \in \operatorname{int} W$. However, from $y^{k} \in f(A)$ for any $k$, it follows that $y^{k^{*}} \in f(A) \subseteq \partial W$, a contradiction. Next, we consider Case 2. If for any $x \in A$ and any $t \geq 0, y^{*} \neq t x$, then, given that $A$ is a standard simplex, there exists $i \in N$ such that $y_{i}^{*}<0$. Since $y^{*}$ is the point to which the sequence $y^{k}$ converges, for some $k^{*}$, we have $y_{i}^{k^{*}}<0$. However, this contradicts that for any $x \in A, f(x) \geq 0$. Hence, $f(A)$ is closed. Given that $f(x)$ is bounded and $A$ is compact, $f(A)$ is bounded. Hence, $f(A)$ is compact, and $f$ has a closed graph, which implies $f$ is UHC. Since $f$ is single-valued, $f$ is continuous.

We define $G: A \rightarrow A$ as

$$
G(x)=\left\{\frac{e^{S}}{|S|} \mid S \subseteq N, S \neq \emptyset, \text { and } f(x) \in \hat{\eta}^{S}(V)\right\} .
$$


Claim 2. $G$ is UHC.

Proof. First, we show that for any $x \in A, G(x) \neq \emptyset$. By the nonemptiness of $f$ and (A.6), $f(x) \in \partial W$ for any $x \in A$. It follows from (A.5) that $f(x) \in \partial W \subseteq W$ and $f(x) \in \bigcup_{S \subseteq N} \hat{\eta}^{S}(V)$. This implies $G(x) \neq \emptyset$.

Next, we show that $G$ is UHC. Let $x^{p} \rightarrow x\left(x^{p} \in A, \forall p\right)$ and $y^{p} \in G\left(x^{p}\right)$ for any $p$. Given that $G(x)$ is finite and compact for every $x \in A$, it suffices to show that if $y^{p} \rightarrow y$, then $y \in G(x)$. The finiteness of $G(x)$ implies that there exists $\hat{p}$ such that $y^{p}=y$ for any $p \geq \hat{p}$. Hence, for any $p>\hat{p}, y \in G\left(x^{p}\right)$. Thus, for any $p>\hat{p}$, there exists a coalition $S^{*}$ such that $y=\frac{e^{S^{*}}}{\left|S^{*}\right|}$ and $f\left(x^{p}\right) \in \hat{\eta}^{S^{*}}(V)$. From the continuity of $f, f\left(x^{p}\right) \rightarrow f(x)$. Moreover, as $\hat{\eta}^{S^{*}}(V)$ is closed, we have $f(x) \in \hat{\eta}^{S^{*}}(V)$. Hence, $y \in G(x)$.

Let $h: A \times A \rightarrow A$ be a function given by, for any $i \in N$,

$$
h_{i}(x, g)=\frac{x_{i}+\max \left\{g_{i}-\frac{1}{n}, 0\right\}}{1+\sum_{j \in N} \max \left\{g_{i}-\frac{1}{n}, 0\right\}} .
$$

Note that $h$ is the same as the function defined by Shapley and Vohra (1991). This function is continuous. We follow their step and define the following function:

$$
h \times \operatorname{co}(G): A \times A \rightarrow A \times A,
$$

namely, $h \times c o(G)(x, g)=\{(h(x, g), c o(G(x)))\} \subseteq A \times A{ }^{* 13}$ Note that $h \times c o(G)$ is convex-valued, compact-valued, and UHC. According to Kakutani's fixed point theorem, there is a fixed point $\left(x^{*}, g^{*}\right) \in$ $A \times A$. More specifically, $x^{*}=h\left(x^{*}, g^{*}\right)$ and $g^{*} \in c o\left(G\left(x^{*}\right)\right)$. Hence, for any $i \in N$, we have

$$
x_{i}^{*}=\frac{x_{i}^{*}+\max \left\{g_{i}^{*}-\frac{1}{n}, 0\right\}}{1+\sum_{j \in N} \max \left\{g_{i}^{*}-\frac{1}{n}, 0\right\}},
$$

or equivalently,

$$
x_{i}^{*}\left(\sum_{j \in N} \max \left\{g_{i}^{*}-\frac{1}{n}, 0\right\}\right)=\max \left\{g_{i}^{*}-\frac{1}{n}, 0\right\} .
$$

Claim 3. $e^{N}=\left(\frac{1}{n}, \ldots, \frac{1}{n}\right) \in \operatorname{co}\left(G\left(x^{*}\right)\right)$.

Proof. As $g^{*} \in \operatorname{co}\left(G\left(x^{*}\right)\right)$, it suffices to show $e^{N}=g^{*}$. Assume that $e^{N} \neq g^{*}$. We have

$$
\sum_{j \in N} \max \left\{g_{i}^{*}-\frac{1}{n}, 0\right\}>0 .
$$

Define $I=\left\{i \in N \mid x_{i}^{*}>0\right\}$ and $K=\left\{k \in N \mid x_{k}^{*}=0\right\}$. Note that $N=I \cup K$ because $x^{*} \in A$. For every $i \in I$,

$$
\max \left\{g_{i}^{*}-\frac{1}{n}, 0\right\} \stackrel{(A .10)}{=} x_{i}^{*}\left(\sum_{j \in N} \max \left\{g_{i}^{*}-\frac{1}{n}, 0\right\}\right) \stackrel{\left(x_{i}^{*}>0\right),(A .11)}{>} 0 .
$$

Hence, $K \neq \emptyset$, because if not, then $g^{*} \in A$ contradicts $g_{i}^{*}>\frac{1}{n}$ for any $i \in I=N$.

\footnotetext{
*13 We denote by $c o(X)$ the convex hull of $X$.
} 
Now, from $g^{*} \in \operatorname{co}\left(G\left(x^{*}\right)\right)$ and (A.12) - i.e., $g_{i}^{*}>\frac{1}{n}>0$ for any $i \in I-$ it follows that for any $i \in I$, there exists $S$ such that $i \in S$ and $f\left(x^{*}\right) \in \hat{\eta}^{S}(V)$. Hence, $f\left(x^{*}\right) \in \partial W \subseteq W \subseteq Q$ implies $f_{i}\left(x^{*}\right)<q$ for any $i \in I$, and we have $f\left(x^{*}\right) \geq 0$. On the other hand, for any $k \in K \neq \emptyset$, we have $f_{k}\left(x^{*}\right)=t x_{k}^{*}=0$ for some $t$. However, this contradicts the second property of $W$.

Now let $\mathcal{T}_{x^{*}}=\left\{S \in 2^{N} \mid f\left(x^{*}\right) \in \hat{\eta}^{S}(V)\right\}$. Since, in general, a collection $\mathcal{B}$ is balanced if and only if $\frac{e^{N}}{n} \in \operatorname{co}\left(\left\{\frac{e^{S}}{|S|} \mid S \in \mathcal{B}\right\}\right)$, it follows from Claim 3 and (A.7) that $\mathcal{T}_{x^{*}}$ is balanced. Hence, we obtain the point $f\left(x^{*}\right)$ and the balanced collection $\mathcal{T}_{x^{*}}$ such that $f\left(x^{*}\right) \in \partial W \cap \mathbb{R}_{+}^{N}$ (by (A.6)) and $f\left(x_{*}\right) \in \cap_{S \in \mathcal{T}_{x^{*}}} \hat{\eta}^{S}(V)$ (by (A.7)).

As we assumed, $V$ is unbindingly balanced. By Definition 4.1, we have

$$
f\left(x^{*}\right) \in V^{+}(N) .
$$

We show that no coalition $S$ unbindingly deviates from $f\left(x^{*}\right)$. For simplicity, let $u^{*}=f\left(x^{*}\right)$. Assume that some coalition $S$ unbindingly deviates from $u^{*}$. By the definition of unbinding deviation, for every $\bar{u}^{S} \in \eta^{S}(V)$, we have $\bar{u}_{j}^{S}>u_{j}^{*}$ for any $j \in S$. We arbitrarily choose and fix $\bar{u}^{S} \in \eta^{S}(V)$. Note that $\bar{u}_{j}^{S}<q$ for any $j \in S$ by the definition of $Q$. As $u^{*}=f\left(x^{*}\right)$, we have $u^{*} \geq 0$ and $u^{*}<q e^{N}$. Now, we define a vector $\bar{u}^{N} \in \mathbb{R}^{N}$ as follows: $\bar{u}_{j}^{N}=\bar{u}_{j}^{S}$ for any $j \in S$ and $\bar{u}_{j}^{N} \in\left(u_{j}^{*}, q\right)$ for any $j \in N \backslash S$. Thus, we have $\bar{u}^{N}>u^{*}$. Moreover, $\bar{u}^{N} \in \hat{\eta}^{S}(V)$ and $\bar{u}^{N}<q e^{N}$, which implies that $\bar{u}^{N} \in W$. As we mentioned, $u^{*}=f\left(x^{*}\right) \in \partial W$. However, in view of the first property of $W$, this contradicts $\bar{u}^{N}>u^{*}$ and $\bar{u}^{N} \in W$.

Finally, we show $C^{U B}(V) \neq \emptyset$. From (A.13), we have $u^{*}=f\left(x^{*}\right) \in V^{+}(N)$, and by the definition of $V^{+}$, there exists $u^{* *} \in V(N)$ such that $u^{* *} \geq u^{*}$. Hence, the fact that no coalition $S$ unbindingly deviates from $u^{*}$ implies that no coalition $S$ unbindingly deviates from $u^{* *}$. Thus, $u^{* *} \in C^{U B}(V)$.

\section{Proof of Proposition 5.3}

Proof. Fix $V$ and write $C^{\mathrm{UB}}$ instead of $C^{\mathrm{UB}}(V)$ for simplicity. First, we show that $C^{\mathrm{UB}}=C C^{\mathrm{UB}}$. It suffices to show that $C^{\mathrm{UB}} \supseteq C C^{\mathrm{UB}}$. We assume that there exists $x \in V(N)$ such that $x \in C C^{\mathrm{UB}}$ and $x \notin C^{\mathrm{UB}}$. From $x \notin C^{\mathrm{UB}}$, it follows that

$$
\text { there exists } \hat{S} \subseteq N \text { such that } \hat{S} \operatorname{dev}^{\mathrm{UB}} x .
$$

In view of the definitions of $\operatorname{dev}^{\mathrm{UB}}$, we have

$$
\text { for any } s \in V(\hat{S}) \text { and any } j \in \hat{S}, s_{j}>x_{j} \text {. }
$$

Now, from $x \in C C^{\mathrm{UB}}$, it follows that for every credible ${ }^{\mathrm{UB}} S, S$ does not unbindingly deviate from $x$. Hence, (A.14) implies that $\hat{S}$ is not credible ${ }^{\mathrm{UB}}$. By the definition of credible $^{\mathrm{UB}}$, it holds that

$$
\text { for any } y \in V(\hat{S}) \text {, there exists credible }{ }^{\mathrm{UB}} T \subsetneq \hat{S} \text { such that } T \operatorname{dev}^{\mathrm{UB}} y \text {. }
$$

In view of the definitions of $\mathrm{dev}^{\mathrm{UB}}$, we have

$$
\text { for any } y \in V(\hat{S}) \text {, there exists a credible }{ }^{\mathrm{UB}} T \subsetneq \hat{S} \text { such that for any } t \in V(T), t_{j}>y_{j} \text {. }
$$


By (A.15), (A.17) implies that

$$
\text { there exists a credible } \mathrm{UB}^{\mathrm{UB}} \subsetneq \hat{S} \text { such that for any } t \in V(T), t_{j}>x_{j} .
$$

Hence, there exists a credible ${ }^{\mathrm{UB}}$ coalition $T$ such that $T \operatorname{dev}^{\mathrm{UB}} x$, which contradicts $x \in C C^{\mathrm{UB}}$.

Next, we show that $C^{\mathrm{UB}}=C C^{* \mathrm{UB}}$ in a similar manner. We replace "for all $y \in V(\hat{S})$ " with "for some $y \in V(\hat{S})$ " in (A.16) and (A.17) (and, of course, $C C^{\mathrm{UB}}$ with $C C^{* \mathrm{UB}}$ and credible ${ }^{\mathrm{UB}}$ with credible*UB throughout the proof). However, (A.18) still holds because (A.15) is valid for all $s \in V(\hat{S})$.

\section{References}

[1] Abe T (2018) Consistency and the core in games with externalities. Int J Game Theory 47:133-154

[2] Abe T, Funaki Y (2017) The non-emptiness of the core of a partition function form game. Int J Game Theory 46:715-736

[3] Aumann RJ (1960) Linearity of unrestrictedly transferable utilities. Naval Research Logistics Quarterly 7:281-284

[4] Bhattacharya A (2004) On the equal division core. Soc Choice Welfare 22:391-399

[5] Funaki Y, Yamato T (1999) The core of an economy with a common pool resource: A partition function form approach. Int J Game Theory 28:157-171

[6] Greenberg J (1994) Coalition structures. Handbook of game theory with economic applications, Chapter 37, 1306-1337, Elsevier

[7] Harsanyi JC, Selten R (1988) A general theory of equilibrium selection in games. MIT Press, Cambridge, Massachusetts

[8] Kóczy L (2007) A recursive core for partition function form games. Theory Decis 63:41-51

[9] Kóczy L and Lauwers L (2004) The coalition structure core is accessible. Games Econ Behav, 48:8693

[10] Konishi H, Quint T, Wako J (2001) On the Shapley-Scarf economy: the case of multiple types of indivisible goods. J Math Econ 35:1-15

[11] Moulin H (2014) Cooperative microeconomics: a game-theoretic introduction. Princeton University Press, Princeton, New Jersey

[12] Peleg B, Sudhölter P (2007) Introduction to the theory of cooperative games. Springer, Berlin, Heidelberg

[13] Predtetchinski A, Herings PJJ (2004) A necessary and sufficient condition for non-emptiness of the core of a non-transferable utility game. J Econ Theory 116:84-92

[14] Ray D (1989) Credible coalitions and the core. Int J Game Theory 18:185-187

[15] Scarf HE (1967) The core of an N person game. Econometrica 35:50-69

[16] Shapley L, Vohra R (1991) On Kakutani's fixed point theorem, the KKMS theorem and the core of a balanced game. Econ Theory 1:108-116

[17] Shapley L, Scarf H (1974) On cores and indivisibility. J Math Econ 1:23-37

[18] Sprumont Y (1990) Population monotonic allocation schemes for cooperative games with transferable utility. Games Econ Behav 2:378-394 
[19] Thrall RM (1961) Generalized characteristic functions for n-person games. Proceedings of the Princeton University Conference of Oct. 1961

[20] Thrall RM, Lucas WF (1963) n-Person games in partition function form. Naval Research Logistics Quarterly 10:281-298

[21] von Neumann J, Morgenstern O (1944) Theory of Games and Economic Behavior. Princeton University Press, Princeton, New Jersey 